\title{
Jahres-Inhalt Vol. 2, 1992
}

\section{Nr. 1}

Editorial

Verhaltenstherapie - Aufgaben, Ziele und Erwartungen erreicht?

Hand, I., Wittchen, H.-U

Tagungen und Kongresse

Impressum

1

Inhaltsverzeichnis 2

Hinweise für Autoren $\quad 110$

Originalarbeiten

Fehlattribution und Erregungstransfer: Eine Studie zur Induktion und Intensivierung von Emotionen

Reinecker, H., Gattinger, E 6

Psychische Beschwerden bei chronischem Tinnitus: Erprobung und Evaluation des Tinnitus-Fragebogens (TF)

Goebel, G., Hiller, W 13

Exposition in-vivo bei sozialen Ängsten und Defiziten

Wlazlo, Z., Schroeder-Hartwig, K., Münchau, N., Kai

ser, G, Hand, 1

23

Aus der Praxis für die Praxis

Strukturierte Information über Vulnerabilität und Belastungsmanagement für schizophrene Patienten

Stark, F.-M 40

Verhaltenstherapeutische Behandlung eines Mann-zu-Frau-

Transsexuellen

Rose, A 48

Gruppen-Verhaltenstherapie in der gesetzlichen Krankenversicherung der BRD. Eine kritische Auseinandersetzung aus

Praxis-Perspektive

Köhlke, H.-U 55

Diskussionsforum

Gemeinsame Stellungnahme der Spitzenverbände der Krankenkassen zum Forschungsgutachten zu Fragen eines Psychotherapeutengesetzes 62

Stellungnahme des Fachverbands klinische Verhaltenstherapie zum Forschungsgutachten zu Fragen eines Psychotherapeutengesetzes 65 
Mitteilungen der Verbände

Ausbildungsinstitut für Klinische Verhaltenstherapie in NRW

e.V. (AFKV) 73

Gesellschaft für Angewandte Psychologie und Verhaltensmedizin mbH (APV) 74

Arbeitsgemeinschaft für Verhaltensmodifikation (Deutschland) (AVM-D) 76

Arbeitsgemeinschaft für Verhaltensmodifikation (Österreich)

(AVM-Ö) $\quad 78$

Centrum für Integrative Psychotherapie (CIP) 81

Deutsche Akademie für Verhaltenstherapie (DAVT) 83

Deutsche Gesellschaft für Verhaltenstherapie (DGVT) .... 84

Fachverband klinische Verhaltenstherapie e.V. (FKV) .... 89

Institut für Fort- und Weiterbildung in Klinischer Verhal

tenstherapie (IFKV) 95

Norddeutsche Pflegeakademie 96

Tübinger Akademie für Verhaltenstherapie (TAVT)

Verein zur Förderung der klinischen Verhaltenstherapie

(VFKV)

101

Vereinigung der Kassenpsychologen

102

Nr.2

Originalarbeiten

Prävention von Ehe- und Partnerschaftsstörungen EPL (Ehe-

vorbereitung - Ein Partnerschaftliches Lernprogramm)

Thurmaier, F., Engl, J., Eckert, V., Hahlweg, K 116

Kognitive und situative Bedingungen des Rückfalls bei Rau-chern Minneker-Hügel, E., de Jong-

Meyer, R., Buchkremer, G. . 125

Affektive Störungen bei Jugendlichen: Prävalenz, Komorbidi-tät und psychosoziale Korrelate

Lewinsohn, P.M., Hops, H., Roberts, R.E., Seeley, J.R.,

Rohde, P., Andrews, J.A., Hautzinger, M 132

Diagnostik von Persönlichkeitsstörungen nach den Kriterien

aktueller internationaler Klassifikationssysteme

Bronisch, T 140

Aus der Praxis für die Praxis

Selbsterfahrung in der Ausbildung zum Verhaltenstherapeu-

ten. Erfahrungsbericht

Görlitz, G, Hippler, B 151

Neuigkeiten vom Buch-und Zeitschriftenmarkt

Mitteilungen der Verbände

Ausbildungsinstitut für Klinische Verhaltenstherapie e.V.

(AFKV)

161

Bayerische Private Akademie für Psychotherapie (BAP) ... 162

Centrum für Integrative Psychotherapie (CIP) 163

Einladung zur 2. Bayerischen Verhaltenstherapietagung des

CIP 1992 168 
Deutsche Akademie für Verhaltenstherapie (DAVT e.V.) . .172

Deutsche Gesellschaft für Verhaltenstherapie (DGVT) .... 174

9. überregionale Workshoptagung der DGVT in Tubingen . 177

Institut für Fort- und Weiterbildung in Klinischer Verhal

tenstherapie (IFKV) 178

Tübinger Akademie für Verhaltenstherapie (TAVT)

Vereinigung der Kassenpsychologen

Tagungen und Kongresse

182

Impressum

113

Inhaltsverzeichnis

114

Hinweise für Autoren $\quad 190$

Nr.3

Editorial

Verluste und Reservekapazitäten im Alter: Ein dynamischer

Kontext der Psychopathologie im Alter

Baltes, M.M 195

Jahres-Inhalt 1992

377

Originalarbeiten

Intellektuelles Selbst-Management im Alter

Skinner, B.F 198

Kognitive Interventionsforschung mit alten Menschen im Be-

reich der fluiden Intelligenz: Grundlagen und Ergebnisse

Sowarka, D 204

Verhaltenstherapie bei Depression im Alter

Hautzinger, M 217

Das Realitätsorientierungstraining - eine spezifische Interven

tion bei Verwirrtheit

Noll, P., Haag, G 222

Depressionsniveau und Belastungsverarbeitung bei Betreuern

körperlich und geistig beeinträchtigter älterer Menschen

Walsh, W.A., Steffen, A., Gallagher-Thompson, D 231

Akzeptanz von verhaltenstherapeutischer Behandlung und Pharmakotherapie bei geriatrischen

Verhaltensstörungen: Ein Vergleich von Experten- und Konsumentengruppen Burgio, L.D.,

Janosky, J.E., Sinnott, J., Hohman, M.J. . . 237

Verhaltenstherapeutische und psychodynamische Therapie-

ansätze bei Altersdepressionen

Fuchs, T., Zimmer, F.T

244

Aus der Praxis für die Praxis

Weiterbildung in Klinischer Psychologie/Psychotherapie - Ein

Bericht über die Einrichtung eines Weiterbildungscurricu-

lums an der Universität Bonn

Scholz, O., Bongers, A

251

Diskussionsforum 
Aktuelle verhaltenstherapeutische Standardprogramme: Moderner Rückschritt in die Symptomtherapie?!

Köhlke, H.-U 256

Kommentar zum Beitrag Köhlke: Aktuelle verhaltensthera peutische Standardprogramme: Moderner Rückschritt in die Symptomtherapie

Fiegenbaum, W 263

Kommentar zum Beitrag Köhlke: Aktuelle verhaltensthera peutische Standardprogramme: Moderner Rückschritt in die Symptomtherapie. Erfahrung, Wissenschaft und Pra xis: Mißverständnisse über empirisch fundierte Psychotherapie

Margraf, J., Schneider, S 265

Neuigkeiten vom Buch- und Zeitschriftenmarkt 267

Mitteilungen der Verbände

Ausbildungsinstitut für Klinische Verhaltenstherapie in NRW

e.V. (AFKV) 269

Gesellschaft für Angewandte Psychologie und Verhaltensme-

dizin mbH (APV GmbH) 270

Arbeitsgemeinschaft für VerhaltensModifikation e.V.

(AVM-D) 271

Centrum für Integrative Psychotherapie (CIP)

Deutsche Akademie für Verhaltenstherapie (DAVT) 274

Deutsche Gesellschaft für Verhaltenstherapie (DGVT) .... 276

Fachverband klinische Verhaltenstherapie e.V. (FKV) .... 279

Österreichische Gesellschaft für Verhaltenstherapie (ÖGVT) 284

Tübinger Akademie für Verhaltenstherapie (TAVT) 285

Vereinigung der Kassenpsychologen 286

Verein zur Förderung der klinischen Verhaltenstherapie

(VFKV) 288

Kurzinformation über die Gründung einer Arbeitsgemein

schaft Ärztliche Verhaltenstherapie (AÄVT) 290

Tagungen und Kongresse 291

Impressum 193

Inhaltsverzeichnis $\quad 194$

Hinweise für Autoren 295

Nr.4

Originalarbeiten

Einfluß von prämorbider Anpassung, sozialen Netzwerkpara-

metern und sozialer Unterstützung auf die Negativsympto-

matik ersterkrankter Schizophrener

Rey, E.-R., Bailer, J., Laubenstein D., Graßhoff, U. . . . 300 Biofeedback der

Stirnmuskelspannung als verhaltensmedi-

zinische Intervention zur Reduktion des Atemwider-

standes?

Maß, R., Ritz, T 309 
Depression und chronische Schmerzen: Zur Bedeutung alltäglicher Belastungen und Handlungskontrolle

Luka-Krausgrill, U., Wurmthaler, C. Wiesheu, M.,

Becker, T 314

HIV-Prävention bei Drogenabhängigen: Ein Therapiepro-

gramm

Fahrner, E.-M., Jürgensmeyer, S., von Törne, 1321

Aus der Praxis für die Praxis

Verhaltenstherapie bei stotternden Kindern: Ein Behandlungs-

paket

Kern, M.C 328

Diskussionsforum

Reizüberflutung: In der Forschung gut belegt, in der Praxis

nicht angewandt? Teil I

Margraf, J 334

Reizkonfrontation: Standardtherapie nur für Standardpatien-

ten?

Schulte, D 335

Konfrontative Behandlung: Erfolg ohne Akzeptanz in der

Praxis

Fiegenbaum, W., Freitag, M., Frank, B 339

Exposition und Reaktionsverhinderung: Ausgewählte Aspekte bei der Behandlung von Zwängen

Reinecker, H 341

Neuigkeiten vom Buch- und Zeitschriftenmarkt 346

Mitteilungen der Verbände

Arbeitsgemeinschaft für Verhaltensmodifikation e.V. (AVM)

Ausbildungsinstitut für Klinische Verhaltenstherapie in NRW

(AFKV) 355

Deutsche Gesellschaft für Verhaltenstherapie e.V. (DGVT) .

Fachverband klinische Verhaltenstherapie e.V. (FKV) .... 365

Institut für Fort- und Weiterbildung in Klinischer Verhaltens

therapie e.V. (IFKV) 367

Tübinger Akademie für Verhaltenstherapie (TAVT)

Verein zur Förderung der klinischen Verhaltenstherapie

(VFKV) 369

Vereinigung der Kassenpsychotherapeuten 370

Tagungen und Kongresse

373

Impressum

297

Inhaltsverzeichnis

Autorenverzeichnis 1992

298

Jahres-Inhalt 1992

375

Sachwortregister 1992

376

Hinweise für Autoren 